\title{
Investigation of Some Properties of KDP Single Crystals Grown by Sankaranarayanan-Ramasamy (SR) Method
}

\author{
Vinh Trung Phan, Anh Quynh Le, Dat Thanh Huynh \\ Faculty of Physics-Engineering Physics, University of Science, Vietnam National University, Ho Chi Minh City, Vietnam
}

Email address:

ptvinh@hcmus.edu.vn (V. T. Phan)

\section{To cite this article:}

Vinh Trung Phan, Anh Quynh Le, Dat Thanh Huynh. Investigation of Some Properties of KDP Single Crystals Grown by SankaranarayananRamasamy (SR) Method. American Journal of Physics and Applications. Vol. 6, No. 1, 2017, pp. 11-17. doi: 10.11648/j.ajpa.20180601.13

Received: September 27, 2017; Accepted: November 1, 2017; Published: December 5, 2017

\begin{abstract}
Potassium Dihydrogen Phosphates (KDP) crystal has been studied since the early 1930s. It has many important applications, such as the electro-optic modulator, Q-switches, the ultrasonic transducer, the shutter for high-speed photography, frequency doubling and high power laser frequency conversion for fusion research. In response to these applications, the highquality KDP single crystals are required. The common method of KDP crystal growth is the lowering of the temperature of saturated KDP solution which is held in a cylindrical tank. The disadvantage of this method is the spontaneous appearance of crystalline clusters at the bottom of the tank which retard the growth of the main crystal. Since the 2010s, Sankaranarayanan and Ramasamy have proposed a new method of growing crystal by designing the Y-shaped solution tank. In this study, the KDP single crystals were grown by the Sankaranarayanan-Ramasamy (SR) method. These crystals have better quality, fewer defects, higher hardness and density, and especially material saving compared to ones from the conventional method.
\end{abstract}

Keywords: KDP, SR Method, Lowering Temperature, Material Saving, Second Harmonic Generation (SHG)

\section{Introduction}

Today, demands for high-quality single crystals in high technologies such as photonics, laser, microelectronics, communications, etc. are increasing. However, single crystals available in nature have not yet met these needs because of their low purity. Therefore, it is necessary to grow single crystals artificially. The techniques used to grow crystals in solution differ mainly in the ways in which the supersaturation is created. Three methods are known: the lowering of the temperature, the evaporation of the solvent, and solution replenishment [1]. The crystal growth from aqueous solution is suitable for water-soluble materials, and their solubility varies widely in temperature, such as KDP $\left(\mathrm{KH}_{2} \mathrm{PO}_{4}\right), \mathrm{ADP}\left(\mathrm{NH}_{4} \mathrm{H}_{2} \mathrm{PO}_{4}\right), \mathrm{KAl}\left(\mathrm{SO}_{4}\right)_{2}$, etc.

Among the single crystals are grown from solution, the KDP crystal is widely researched since it can be easily grown to large sizes. Two important properties of KDP crystal studied extensively are the second harmonic generation (SHG) and Pockels effect. The SHG is the three-wave mixing process which a laser radiation frequency $\omega$ spreads through a crystal and interacts with the environment and itself. The result is the appearance of the double frequency, $2 \omega$. The
SHG can be applied in systems of frequency laser conversion in the laboratory, such as Sum Frequency Generation (SFG), Difference Frequency Generation (DFG), Optical Parametric Amplifier (OPA), Optical Parametric Oscillator (OPO), etc. [2]. The Pockels effect is from the piezoelectric property of the KDP crystal. When an external electric field is applied along the optical axis of the crystal, its refractive index ellipsoid is deformed, that changes optical properties of the crystal. The Pockels effect is applied to fabricate the phase shifter, the light intensity modulator, and the optical switch.

To date, two trends are considered to improve the quality of KDP crystal and enhance its characteristic properties. Many works have studied the doping of various organic and inorganic substances into KDP crystal, among them $\mathrm{KCl}$ and EDTA (Ethylenediaminetetraacetic Acid). Most commercial chemicals contain heavy metal ions such as $\mathrm{Cr}^{3+}, \mathrm{Fe}^{3+}$, and $\mathrm{Al}^{3+}$. These impurities make defects in crystal lattice structure. The presence of the chlorine anion ( $\mathrm{KCl}$ additive) or two amines and four carboxylates (EDTA additive) in solution reduces effectively heavy metal ions diffusing into the crystal lattice, which improves the quality of crystal [3, 4]. Meanwhile, K. Sankaranarayanan and P. Ramasamy proposed a new method of crystal growth, called the SR 
method. The SR method is a suitable method to grow large single crystals, the habit of which is unidirectional, high transparency and no defects [5]. The entire quantity of the solute was converted into the crystal, thus achieving a solutecrystal conversion efficiency of $100 \%$ [6]. Ramasamy et al. $[6,7]$ reported the KDP crystals grown by the SR method had damage thresholds higher than the conventional method grown crystals. The damage threshold was influenced by the dislocation in the KDP crystal, and the crystal with many dislocations presented low damage threshold [8]. These conclusions showed that the quality of the crystal grown by the SR method is better than conventional method grown crystal for optical device [9].

In this study, single KDP crystals were grown by the temperature lowering method and SR technique with lowcost self-designed crystallization system. The best quality crystals were characterized by Vicker hardness, UV-Vis spectroscopy, FT-Raman analysis, X-Ray diffraction and second harmonic generation effect (SHG). The results showed the superiority of new method.

\section{Method}

\subsection{Material}

KDP was purchased from Guangdong Guanghua Chemical Factory Co., Ltd. (purity $\geq 99,5 \%$ ). The solubility of KDP in water is determined by the formula:

$$
m_{K D P / 100 m l}^{t^{0} C}=\frac{100 \cdot c_{0}}{1-c_{0}}
$$

where $c_{0}=0.116+0.00335 . t^{0}[10]$

\subsection{Experiment}

KDP powder was dissolved in distilled water to form a saturated solution at $50^{\circ} \mathrm{C}$. This solution was filtered three times to remove almost the impurities and dirt. Then the $\mathrm{KDP}$ solution was poured into $\mathrm{Y}$-shaped tanks and annealed at $55^{\circ} \mathrm{C}$ for 24 hours. The process of annealing helped release small air bubbles from the solution and stabilized the temperature of the solution.

A KDP seed (about $2 \times 2 \times 12 \mathrm{~mm}$ ) was attached at the bottom of the solution tank. The tank was put into the system of crystal growth (Figure 2). The tanks were surrounded by water environment which stabilized the temperature of KDP solutions. The temperature of solutions would be lowered $1{ }^{\circ} \mathrm{C}$ /day by a chiller and ranged from $49^{\circ} \mathrm{C}$ to $19^{\circ} \mathrm{C}$. After 30 days, KDP crystals were removed from the tanks (Figure 2(a) and 2(b)). They were cut into $6 \mathrm{~mm}$ thick slices whose both opposite sides were polished (Figure 2(c) and 2(d)).

KDP crystals were also grown by the conventional temperature lowering method in conditions like SR KDP crystals. The seed crystal was hung in the solution. During crystal growth, the appearance of spontaneous nucleation retarded the growth of main single KDP crystal. Polycrystalline clusters at the bottom of the tank increased as time passed. Therefore, the growth rate and size of conventional KDP crystals were limited.
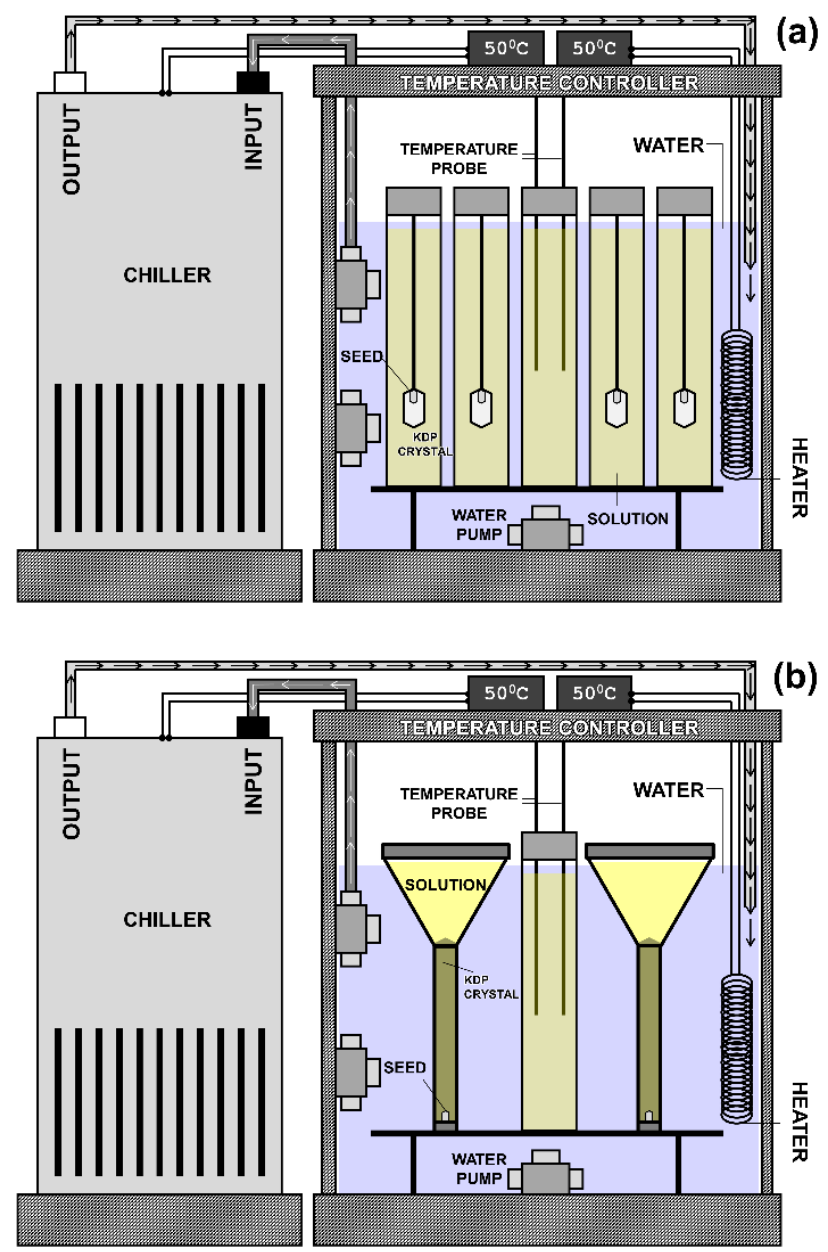

Figure 1. Schematic of the systems of growing KDP crystal by conventional method (a) and the SR method (b).

\section{Results and Discussion}

\subsection{Observation with the Naked Eye}

The number of high-quality KDP crystals by the SR method was more than by conventional method. During the growing process of crystal by the SR method, when small defects (or cracks) occurred somewhere in the crystal, the rest of the crystal remained clear and unaffected. On the other hand, in the conventional method, defects would become increasingly widespread throughout the crystal.

Also, a superior advantage of the SR method over conventional method is that there is no material loss due to spontaneous crystallization (which occurs at the bottom of solution tank in conventional method). All solute accumulated on the crystal seed. Therefore, there was an only growth of a single crystal.

The mass of the largest conventional and SR method grown single KDP crystals was 217.65 and 589.83 grams, respectively.

\subsection{Vicker Hardness}

Hardness is an important factor of a crystal for fabrications 
of optical devices. Vicker hardness studies were carried out by the Nanovea ST500 Mechanical Tester. This tester used a diamond pyramidal indenter for varying loads. The indenter was applied on the (001) face of the KDP crystal for 10 seconds. The Vicker hardness number was displayed on the screen of the tester. The results are shown in Table 1 and Figure 3.

Table 1. Vicker hardness of $S R$ and conventional method grown KDP crystals.

\begin{tabular}{|c|c|c|c|c|}
\hline \multirow[t]{2}{*}{ Sample } & \multirow{2}{*}{$\begin{array}{l}\text { Load } \\
(\mathrm{mN})\end{array}$} & \multicolumn{2}{|c|}{$\begin{array}{l}\text { The diagonal length of } \\
\text { dent }(\mu \mathrm{m})\end{array}$} & \multirow{2}{*}{$\begin{array}{l}\text { Vicker } \\
\text { hardness } \\
\text { (HV) }\end{array}$} \\
\hline & & $\mathbf{D}_{1}$ & $\mathbf{D}_{2}$ & \\
\hline Conventional & 2942 & 78.3 & 74 & 99.23 \\
\hline method grown & 4903 & 90.25 & 92.32 & 115.11 \\
\hline KDP crystal & 9807 & 125.8 & 128.3 & 118.86 \\
\hline The SR method & 2942 & 67.6 & 69.35 & 122.76 \\
\hline grown KDP & 4903 & 83.35 & 88.1 & 130.53 \\
\hline crystal & 9807 & 115.2 & 118.5 & 140.52 \\
\hline
\end{tabular}

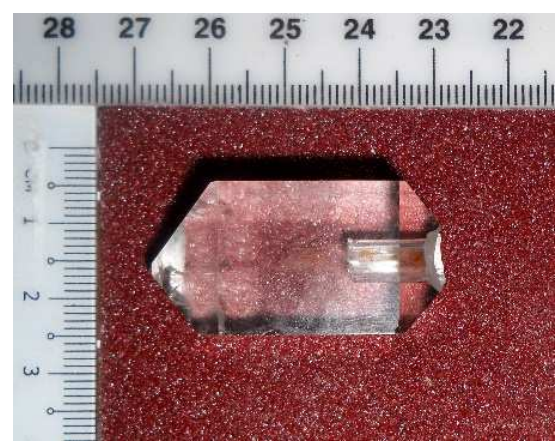

(a) The KDP crystal grown by conventional method

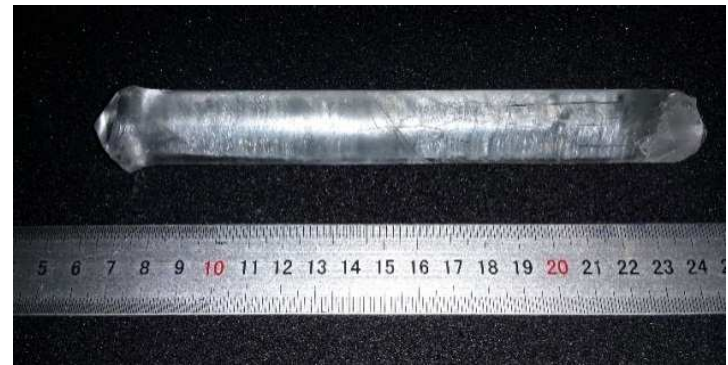

(b) The KDP crystal grown by the SR method

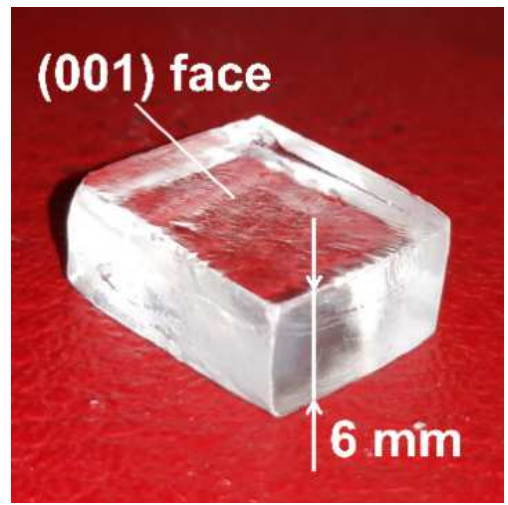

(c) The conventional method KDP crystals after being cut and polished (cross-section perpendicular to the optical axis of KDP crystal or the fourfold inversion axis of KDP crystal)

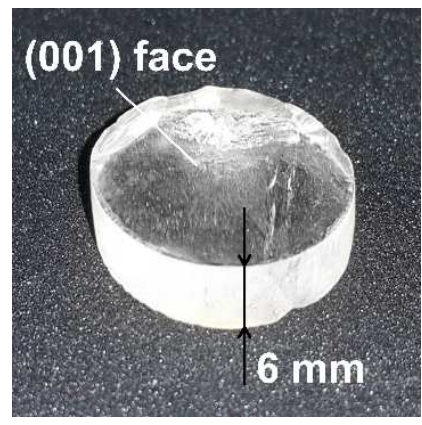

(d) The SR method KDP crystals after being cut and polished (cross-section perpendicular to the optical axis of KDP crystal or the fourfold inversion axis of KDP crystal)

Figure 2. Some samples of KDP crystal.

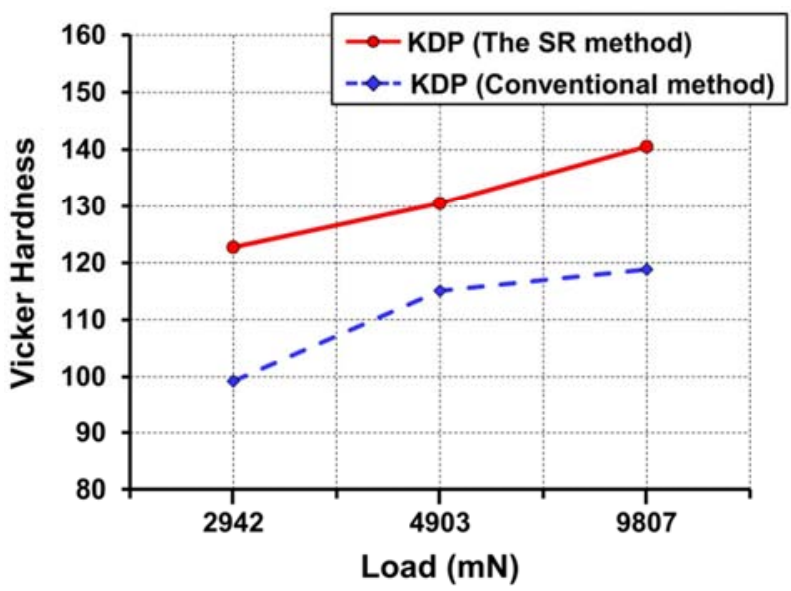

Figure 3. Vicker hardness vs. load for the SR and conventional method KDP crystals.

The measurement showed that when the applied load was $4903 \mathrm{mN}$, the dent was a perfect rhombus in shape. Therefore, the Vicker hardness value at $4903 \mathrm{mN}$ is the most reliable. The SR method grown KDP crystal is harder than the conventional method grown KDP crystal about $15.42 \%$. This result is relatively consistent with Ramasamy's work [7].

\subsection{UV-Vis Spectroscopy}

The transmission and absorption spectra were recorded in a wavelength range from 200 to $1100 \mathrm{~nm}$ using HALO RB10 Dynamica UV-Vis spectrometer. It is observed that the transmittance percentage of the SR method grown crystal is higher than that of conventional method grown crystal. The result can be explained due to unidirectional crystal growth which reduces impurities effectively diffusing into the crystal lattice.

Besides, absorption spectra of the SR and conventional method KDP crystals were analyzed to estimate the band gap energy (Figure 6). KDP crystal is a direct transition material $[4,10]$. Its absorption coefficient $\alpha$ and gap energy $E_{g}$ is given by:

$$
\alpha=\frac{A\left(h v-E_{g}\right)^{\frac{1}{2}}}{h v}
$$

The calculated values of the SR and conventional method 
KDP crystal's band gap energy were 4.97 and $4.67 \mathrm{eV}$, respectively. The band gap is a major factor in understanding the Laser-Induced Damage Threshold (LIDT) of dielectric nonlinear crystals [10]. (LIDT is a physical characteristic of optical component which defines a critical power of laser irradiation causing irreversible changes in materials structure). These values are different from other studies. For example, Robert et al. [11] used the modified SR method for unidirectional growth to grow KDP crystals of $65 \mathrm{~mm}$ length with a period of 13 days. The crystals had a band gap of 3.20 eV. Kucheyev et al. [12] determined the band gap to be close $7.6 \mathrm{eV}$. The different conditions of crystal growth probably resulted in absorption edge deviations.

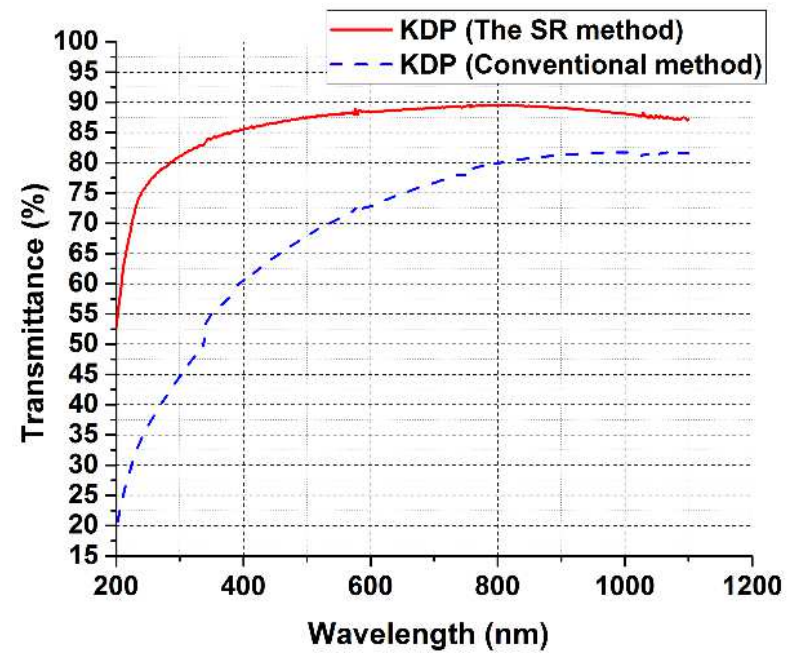

(a) The transmission spectra

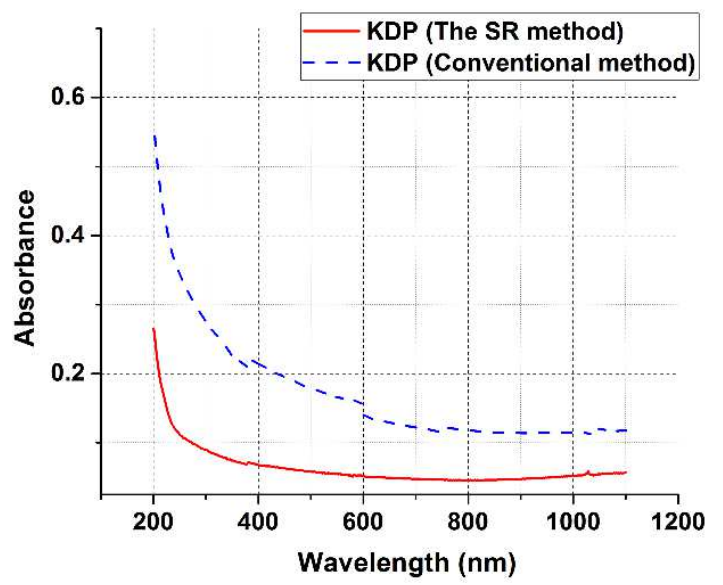

(b) The absorption spectra

Figure 4. UV-Vis spectroscopy of the SR and conventional method KDP crystals.

\subsection{XRD Analysis}

X-ray diffraction studies were carried out for the SR and conventional method KDP crystals. Figure 5 shows the comparison of XRD pattern of KDP samples. It is seen that the SR method grown KDP crystal shows the higher value in peak intensities. Dislocation density or defects is less in crystals grown by SR method. The KDP crystals grown by
SR method have excellent crystalline perfection.

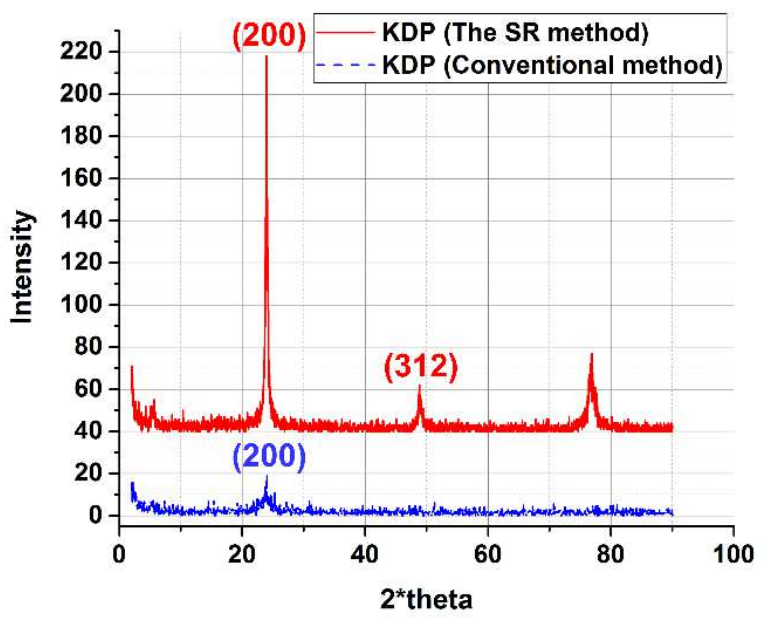

Figure 5. XRD analysis of the $S R$ and conventional method KDP crystals.

\subsection{FT-Raman Analysis}

FT-Raman analysis are essential to understand the various functional groups and structure of a compound. The frequencies of vibrational bonds are shown in Table 2. FTRaman analysis was recorded by LABRAM 300 Horiba JOBIN YVON spectrometer.
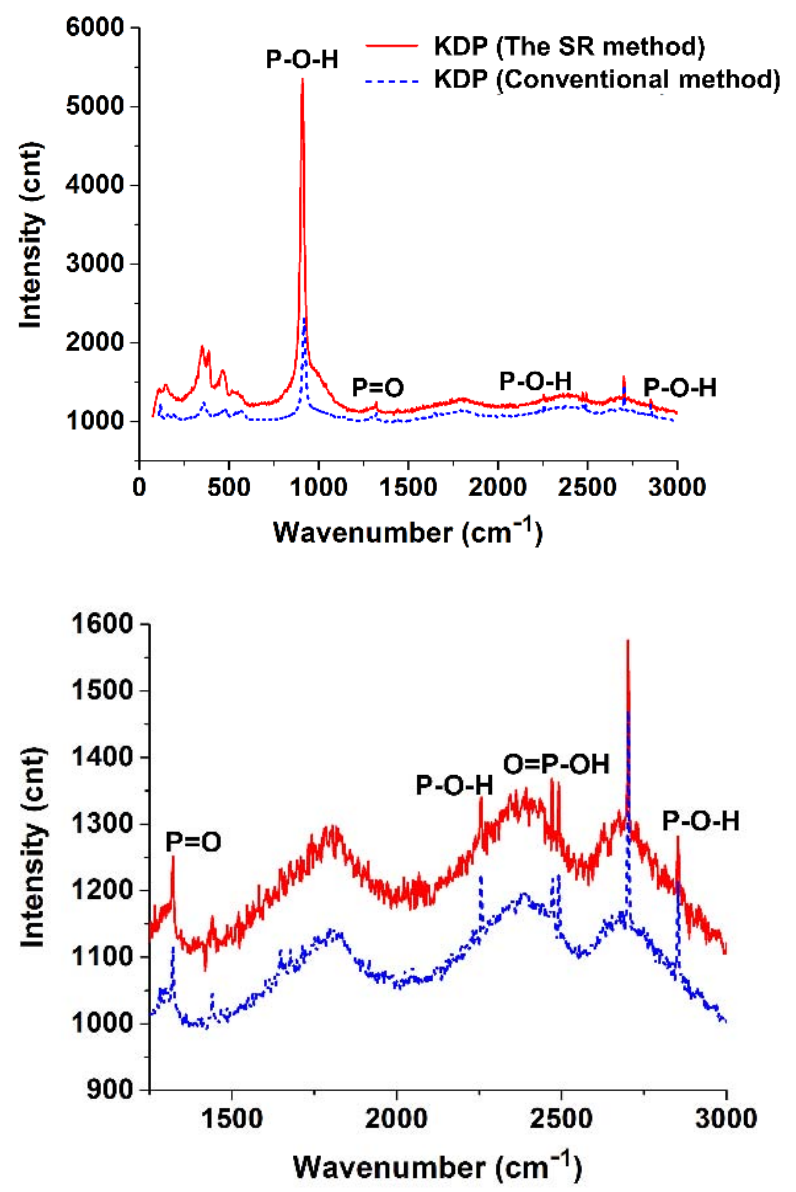

Figure 6. FT-Raman spectra of SR and conventional method KDP crystals. 
Table 2. Observed characteristic Raman frequencies of KDP crystal.

\begin{tabular}{lll}
\cline { 1 - 2 } $\begin{array}{lll}\text { Wavenumber }\left(\mathbf{c m}^{-1}\right) \\
\text { KDP method }\end{array}$ & $\begin{array}{l}\text { Conventional } \\
\text { method KDP }\end{array}$ & Assignments [13] \\
\hline $520(\mathrm{~m})$ & $571(\mathrm{~m})$ & HO-P-OH bending \\
$909(\mathrm{vs})$ & $915(\mathrm{vs})$ & P-O-H stretching \\
$1266(\mathrm{vw})$ & $1281(\mathrm{vw})$ & - \\
$1300(\mathrm{w})$ & $1300(\mathrm{w})$ & $\mathrm{P}=\mathrm{O}$ stretching \\
$1324(\mathrm{vw})$ & $1319(\mathrm{vw})$ & - \\
$1440(\mathrm{vw})$ & $1440(\mathrm{vw})$ & - \\
$1484(\mathrm{vw})$ & $1485(\mathrm{vw})$ & - \\
$2260(\mathrm{w})$ & $2253(\mathrm{w})$ & P-O-H bending \\
$2473(\mathrm{w})$ & $2471(\mathrm{w})$ & $\mathrm{O}=\mathrm{P}-\mathrm{OH}$ stretching \\
$2490(\mathrm{w})$ & $2492(\mathrm{w})$ & - \\
$2703(\mathrm{~m})$ & $2702(\mathrm{~m})$ & - \\
$2851(\mathrm{w})$ & $2854(\mathrm{w})$ & P-O-H asymmetric stretching \\
vs - very strong; - strong; $\mathrm{m}-$ medium; - - weak; vw - very weak \\
\hline
\end{tabular}

Figure 6 shows the FT-Raman spectra of the KDP samples recorded at room temperature in the frequency range of 0 $3000 \mathrm{~cm}^{-1}$. The characteristic peaks of the SR method grown KDP crystal have higher intensities than that of conventional method grown KDP crystal. The present Raman study indicates the advantage of SR method for the crystal structure of KDP, which leads to more bond density. In addition, the wavenumber of all Raman peaks of both method grown KDP crystal is the same. Ramasamy et al. [6] concluded that the constraint growth in the SR method experiment did not affect the crystalline perfection.

FT-Raman analysis can evaluate the nonlinear optical properties of KDP crystal. The anion groups $\left(\mathrm{PO}_{4}\right)^{-3}$ contribute approximately $99 \%$ to SHG coefficient, and cation $\mathrm{K}^{+}$has nothing to do with the SHG effect, and hydrogen bonds contribute much to birefringence [14].

\subsection{Second Harmonic Generation (SHG)}

The best quality KDP crystals were chosen to cut to the type I phase-matching angles $\left(41.1^{\circ}\right)$ [15]. These unidirectional phase-matching crystals were investigated SHG effect by the Nd: YAG laser whose wavelength of 1064 $\mathrm{nm}$ and maximum power of $1 \mathrm{~W}$.

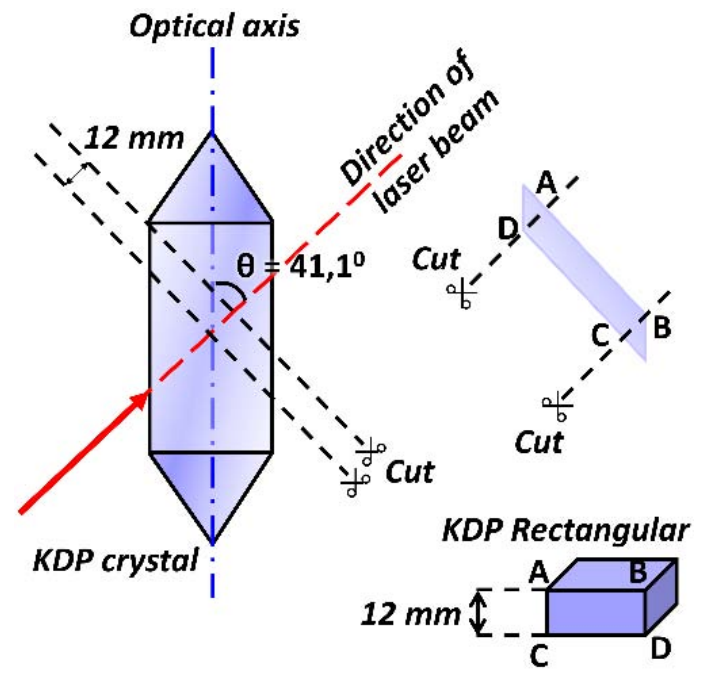

Figure 7. Illustrations to cut KDP crystal to the phase-matching angle.

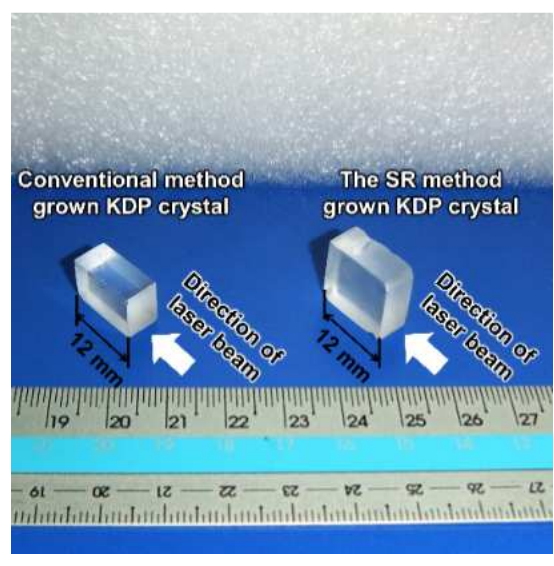

Figure 8. Phase-matching KDP crystal samples.

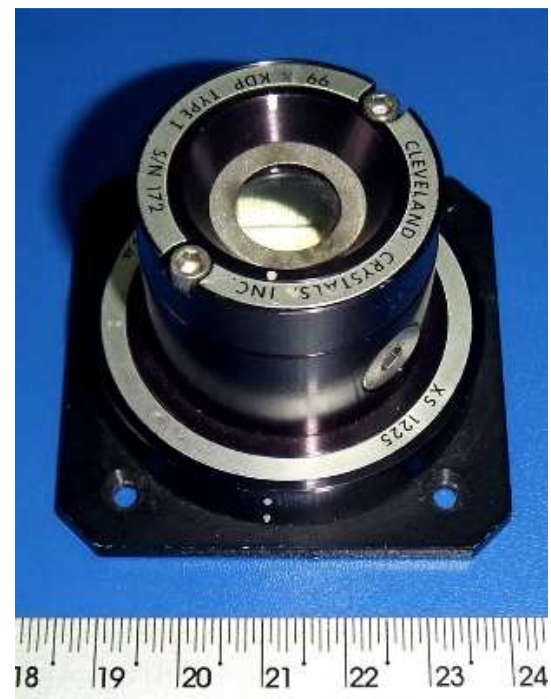

Figure 9. The commercial unidirectional phase-matching KDP crystal.

The optical system of investigating SHG effect included $\mathrm{Nd}$ : YAG laser, sensor card for $1064 \mathrm{~nm}$, an optical filter (rejection wavelength of $1064 \mathrm{~nm}$ ), digital laser power meter, and some other optical components (Figure 10 and Table 3). Also, a commercial unidirectional phase-matching KDP crystal was also prepared to compare with KDP samples in the present work (Figure 9).

According to the theoretical expression, the relationship between the power of $1064 \mathrm{~nm}$ laser and the maximum SHG efficiency is completely linear, and dependence of power of $532 \mathrm{~nm}$ light on the power of $1064 \mathrm{~nm}$ laser is a half of a parabola curve [16]:

$$
\begin{aligned}
e_{S H G}^{\text {max }} & =\frac{P_{532}^{\text {out }}}{P_{1064}^{\text {in }}}=\frac{8}{\pi c}\left(\frac{\mu_{0}}{\varepsilon_{0}}\right)^{\frac{3}{2}} \frac{\omega^{3} d^{2} L}{n^{2}} P_{1064}^{\text {in }} \\
\Rightarrow P_{532}^{\text {out }} & =\frac{8}{\pi c}\left(\frac{\mu_{0}}{\varepsilon_{0}}\right)^{\frac{3}{2}} \frac{\omega^{3} d^{2} L}{n^{2}} P_{1064}^{\text {in }}{ }^{2}
\end{aligned}
$$

where $\mathrm{c}$ is the speed of light in vacuum, $\varepsilon_{0}$ is vacuum permittivity, $\mu_{0}$ is vacuum permeability, $\omega$ is the angular frequency of input laser, $d$ is nonlinear coupling coefficient of crystal, $\mathrm{L}$ is the length of crystal and $\mathrm{n}$ is the refractive index of crystal with the wavelength of input laser. 


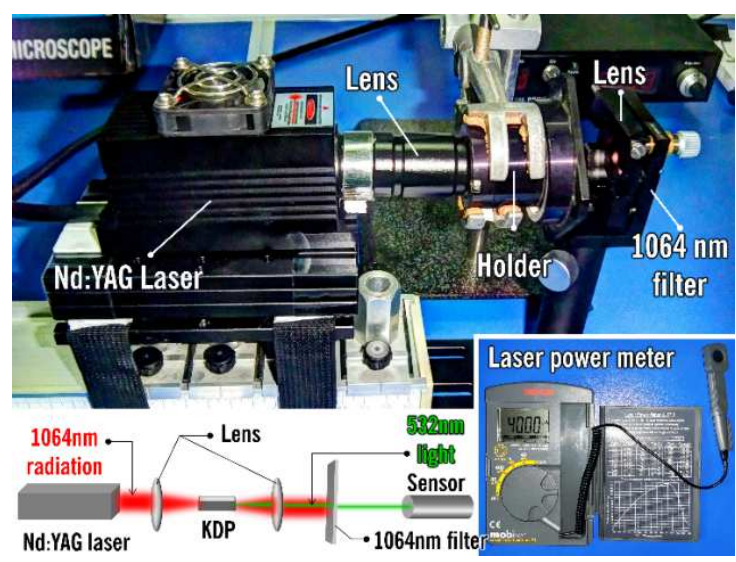

Figure 10. The optical system of investigating SHG effect.

Table 3. The main components of SHG optical system.

\begin{tabular}{lll}
\hline Component & $\begin{array}{l}\text { Model / } \\
\text { Manufacturer }\end{array}$ & Information \\
\hline $\begin{array}{l}\text { Nd: YAG } \\
\text { laser }\end{array}$ & $\begin{array}{l}\text { LSR-PS-II/Lilly } \\
\text { Electronics }\end{array}$ & $\begin{array}{l}\text { Continuous mode; Wavelength of } 1064 \\
\text { nm; Maximum power of } 1 \mathrm{~W}\end{array}$ \\
$1064 \mathrm{~nm}$ & Omega & $\begin{array}{l}\text { Diameter of } 2 \mathrm{~cm} \text {; Thickness of } 2 \mathrm{~mm} \text {; } \\
\text { Transmittance at } 1064 \mathrm{~nm} \text { is } 0.02 \%\end{array}$ \\
$\begin{array}{l}\text { Laser power } \\
\text { meter }\end{array}$ & LP1/Sanwa & Wavelength range from 400 to 1100 \\
Commercial & XS & nm; Minimum difference is $0.01 \mu \mathrm{W}$ \\
KDP crystal & $1224 /$ Cleveland & Type I phase-matching angle; Length of \\
\hline
\end{tabular}

Figure 11 and 12 show the results are suitable for the theoretical expression (3) and (4), respectively.

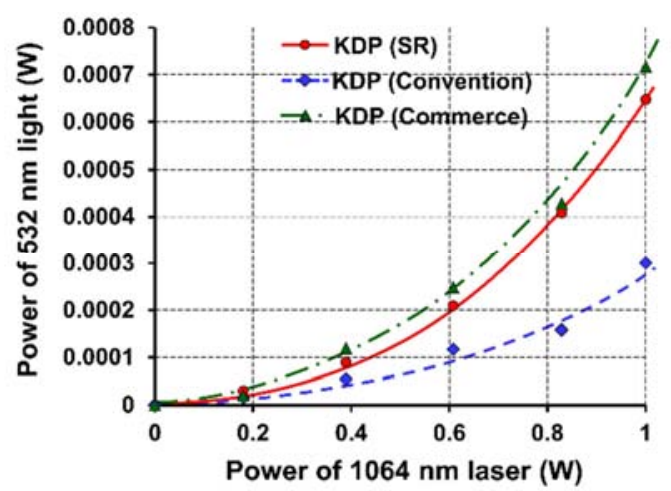

Figure 11. Dependence of power of $532 \mathrm{~nm}$ light on power of 1064nm laser.

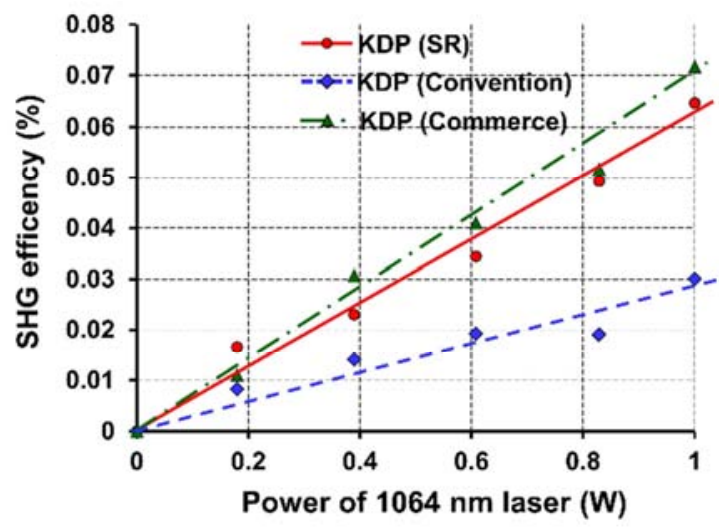

Figure 12. Dependence of SHG efficiency on power of 1064nm laser.
Figure 12 shows that the SHG efficiencies of the SR method grown KDP crystal are higher than that of conventional crystal at laser power values of 0.179 to $1 \mathrm{~W}$. Typically at $1 \mathrm{~W}$ power, the SHG efficiency of the SR method grown KDP crystal is about $122 \%$ greater than the conventional method grown KDP crystal, and approximately near that of commercial crystal (about 93\%). These results showed the superiority of the SR method in enhancing crystal quality.

The SHG conversion efficiencies of the SR method grown KDP crystal have not reached the values of commercial KDP crystal because of several reasons, among them mainly the limitation of surface polishing and lack of AR (antireflective) coating.

\section{Conclusions}

Single KDP crystals have been grown by the temperature lowering method and SR technique. The properties of crystal were investigated by Vicker hardness measurement, UV-Vis spectroscopy, X-ray diffraction, FT-Raman analysis and second harmonic generation (SHG).

The crystal grown by SR method is found mechanically harder and with higher optical transmission than the crystal grown by the conventional method. The XRD study indicates that the SR KDP crystal has good crystalline perfection compared to conventional KDP crystal. These conclusions are relatively consistent with Ramasamy's works $[6,7,8,9]$ and Barati's work [17].

In particular, the SR KDP crystal in the present work has SHG efficiency reached about $93 \%$ of the efficiency value of the commercial KDP crystal which was grown under hightech conditions. This result will be useful to enhance quality of KDP crystal for various applications

At present, the SR method has a lot of potential for the growth of large single crystals with high crystalline perfection and cost saving. It must also be compatible with doping of various organic and inorganic substances into KDP crystal. The research will develop widely in the future.

\section{References}

[1] L. N. Rashkovish, KDP-family Single Crystals, Hilger Ed., Bristol, 166-167 (1991).

[2] Robert W. Boyd, Nonlinear Optics, Academic Press, London (2008).

[3] Guohui Li, Liping Xue, Genbo Su, Zhengdong Li, Xinxin Zhuang, Youping He, Rapid growth of KDP crystal from aqueous solutions with additives and its optical studies, Cryst. Res. Technol, 40, 9, 867-870 (2005).

[4] Anisur Rahman, Jiban Podder, Effect of EDTA on the Growth Kinetics and Structural and Optical Properties of KDP Crystal, International Journal of Optics, Article ID 978763 (2010).

[5] K. Sankaranarayanan, P. Ramasamy, Unidirectional seeded single crystal growth from solution of benzophenone, Journal of Crystal Growth, 280 (3-4), 467-473 (2005). 
[6] N. Balamurugan, P. Ramasamy, Investigation of the Growth Rate Formula and Bulk Laser Damage Threshold KDP Crystal Growth from Aqueous Solution by the SankaranarayananRamasamy (SR) Method, Crystal Growth \& Design, 6 (7), 1642-1644 (2006).

[7] P. Rajesha, S. Sreedhar, K. Boopathi, S. Venugopal Rao, P. Ramasamy, Enhancement of the crystalline perfection of $<00$ $1>$ directed KDP single crystal, Current Applied Physics, 11 (6), 1343-1348 (2011).

[8] S. Balamurugan, P. Ramasamy, S. K. Sharma, Yutthapong Inkong, Prapun Manyum, Investigation of SR method grown $<001>$ directed KDP single crystal and its charaterization by high-resolution X-ray diffractometry (HRXRD), laser damage threshold, dielectric, thermal analysis, optical and hardness studies, Materials Chemistry and Physics, 117 (2-3), 465-470 (2009).

[9] S. Balamurugan, P. Ramasamy, Bulk growth of $<101>$ KDP crystal by Sankaranarayanan-Ramasamy method and its characterization, Materials Chemistry and Physics, 112 (1), 14 (2008).

[10] A. Dyan, G. Duchateau, S. Eslava, J. L. Stehle, D. Damiani, H. Piombini, Transmission measurements in rapid growth KDP and DKDP crystals, Journal of Modern Optics, 56 (1), 45-49 (2009).

[11] R. Robert, C. Justin Raj, S. Krishnan, S. Jerome Das, Growth, theoretical and optical studies on potassium dihydrogen phosphate (KDP) single crystals by modified Sankaranarayanan-Ramasamy (mSR) method, Physica B, 405, 20-24 (2010).

[12] S. O. Kucheyev, C. Bostedt, T. van Buuren, T. M. Willey, T. A. Land, L. J. Terninello, T. E. Felter, A. V. Hamza, S. G. Demos, A. J. Nelson, Electronic structure of KD2xH2(1$\mathrm{x}) \mathrm{PO} 4$ studied by soft x-ray absorption and emission spectroscopies, Phys. Rev. B, 70 (2004).

[13] P. Kumaresan, S. Moorthy Babu, P. M. Anbarasan, Growth and characterization of metal ions and dyes doped KDP single crystals for laser applications, Materials Research Bulletin, 43, 1716-1723 (2008).

[14] Zheshuai Lin, Zhizhong Wang, Chungtian Chen, Mechanism of linear and nonlinear optical effects of KDP and urea crystals, Journal of chemical physics, 118 (5) (2003).

[15] Ruifeng Su, Haitao Liu, Yingchun Liang, Fuli Yu, Residual thermal stress of a mounted KDP crystal after cooling and its effects on second harmonic generation of a high-averagepower laser, Optics \& Laser Technology, 87, 43-50 (2017).

[16] Lan Xinju, Laser Technology, CRC Press, Florida, 207-208 (2010).

[17] F. Barati, H. Rezagholipour Dizaji, Growth of KDP single crystal in second harmonic direction by modified Sankaranarayanan-Ramasamy method, Opt Quant Electron, 48: 432 (2016). 